\title{
El diclofenac y la terapia de manipulación espinal no agregan beneficio al tratamiento habitual del dolor lumbar agudo
}

\author{
Diclofenac and spinal manipulation do not add benefits to usual care in acute lumbar pain
}

\section{Objetivo}

Evaluar si el agregado de diclofenac y/o terapia de manipulación espinal (TME) al tratamiento habitual (paracetamol más consejo) acortan el tiempo de recuperación de los pacientes con dolor lumbar agudo (DLA).

\section{Diseño y lugar y pacientes}

Ensayo clínico factorial ${ }^{*}$ aleatorizado, multicéntrico, doble ciego y controlado con placebo, con un seguimiento de 12 semanas llevado a cabo en 14 centros de atención primaria de Sydney, Australia.

Fueron incluidos 240 pacientes (60 por rama) que habían visitado a su medico de atención primaria por una lumbalgia de menos de seis semanas de duración (DLA) y que referían dolor moderado y moderada discapacidad. El promedio de edad fue 40,7 años, el $44 \%$ era de sexo femenino y la intensidad del dolor, de 6,5 en una escala de uno a diez. No fueron incluidos individuos con dolor lumbar crónico, sospecha de enfermedad espinal o compromiso de una raíz nerviosa, ni quienes estuvieran tomando antiinflamatorios o realizando manipulación espinal o quienes presentaran contraindicaciones al diclofenac, al paracetamol o al TME.

\section{Intervención y medición de los resultados}

Todos recibieron los cuidados habituales (paracetamol más consejo) y se los aleatorizó a cuatro grupos que agregaron: 1) TME más diclofenac; 2) simulación de TME más diclofenac; 3) TME más placebo ; y 4) simulación de TME más placebo. Los resultados fueron registrados al inicio y a la primera, segunda cuarta y duodécima semana. Los primarios fueron el tiempo al primer día sin dolor y hasta pasar siete días consecutivos con un dolor menor a dos en diez. Los secundarios fueron la intensidad del dolor, la funcionalidad, la discapacidad $\mathrm{y}$ el efecto global percibido por el paciente.

\section{Resultados principales}

El $99 \%$ de los pacientes ya estaba recuperado o había sido

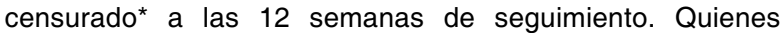
tomaron diclofenac no tuvieron una recuperación más rápida que quienes tomaron placebo (HR 1,09; IC95\% 0,84 a 1,42) ni tampoco la tuvieron quienes recibieron TME real versus TME simulada (HR 1,01; IC95\% 0,77 a 1,31). Tampoco fue menor el tiempo de recuperación en el grupo que recibió ambos tratamientos activos (diclofenac y TME). No hubo diferencias en los resultados secundarios ni en la adherencia a los tratamientos.

\section{Conclusiones}

Los pacientes con DLA que reciben cuidados de primera línea (reposo, paracetamol y educación) tardan el mismo tiempo en recuperarse que aquellos a quienes se les agrega diclofenac y/o TME.

Palabras clave: dolor lumbar, paracetamol, diclofenac, terapia de manipulación espinal, ensayo clínico aleatorizado doble ciego.

Key words: lumbar pain, acetaminophen, diclofenac, spinal manipulation therapy, randomized clinical trial, double blind.

Fuente de financiamiento: Australia's National Health and Medical Research Council. El Diclofenac active fue donado por Alphapharm y las tabletas (Diclofenac y Placebo) fueron producidas por Bill Rae.

\section{Comentario}

Este estudio demuestra que una de las mejores estrategias para el tratamiento del DLA no complicado sería el consejo, el reaseguro y el paracetamol, durante hasta ocho a 12 semanas. Vale destacar que para lograr un adecuado manejo de los pacientes con DLA es necesario descartar patologías complicadas (infecciones, síndromes radiculares, fracturas vertebrales, etc.) y tener un bajo umbral para detectar sus signos de alarma $^{1}$, así como contar con una buena relación medicopaciente y con tiempo para poder informarlo y educarlo.

Siendo el DLA una patología tan prevalente, tendríamos que estar un poco mejor entrenados en identificar y realizar los diagnósticos diferenciales ante este motivo de consulta; por ejemplo para poder discriminar entre aquellos que presentan patología discal y quienes sufren un síndrome miofascial, ya que requieren abordajes diferentes.

\section{Conclusiones de los comentadores}

En los pacientes con DLA es importante pesquisar signos de alarma que nos hagan sospechar otras patologías asociadas; $y$ ante su ausencia, tratar de identificar la causa, dar reaseguro y educación al paciente, e indicar hasta un gramo de paracetamol cada seis horas y terapia específica de alguna eventual causa tratable. Recordamos que hasta el $90 \%$ de los casos se resuelven en menos de ocho semanas.

Ver glosario*

Daniel Weissbrod y Eduardo Stonski [ Grupo de Alivio al Dolor en el Anciano, Programa de Medicina Geriátrica del Servicio de Clínica Médica, Hospital Italiano de Buenos Aires. daniel.weissbrod@hospitalitaliano.org.ar ]

Weissbrod D, Stonski E. El diclofenac y la terapia de manipulación espinal no agregan beneficio al tratamiento habitual del dolor lumbar agudo. Evid. actual. práct. ambul; 11(6): 171. Nov-Dic 2008. Comentado de: Hancock M y col. Assessment of diclofenac or spinal manipulative therapy, or both, in addition to recommended first-line treatment for acute low back pain: a randomised controlled trial. Lancet. 2007 Nov 10;370(9599):1638-43. Disponible en URL: http://www.thelancet.com/journals/lancet/article/PIIS0140-6736(07)61686-9/fulltext\#article_upsell PMID: 17993364

\section{Referencia}

1. Koes B y col. Acute low back pain. Am Fam Physician. 2006 Sep 1; 74 (5):803-5 